\title{
THE SPECTRUM OF THE HODGE LAPLACIAN FOR A DEGENERATING FAMILY OF HYPERBOLIC THREE MANIFOLDS
}

\author{
JÓZEF DODZIUK AND JEFFREY MCGOWAN
}

\begin{abstract}
We consider a sequence $\left(M_{n}\right)_{n=1}^{\infty}$ of compact hyperbolic manifolds converging to a complete hyperbolic manifold $M_{0}$ with cusps. The Laplace operator acting on the space of $L^{2}$ differential forms on $M_{0}$ has continuous spectrum filling the half-line $[0, \infty)$. One expects therefore that the spectra of this operator on $M_{n}$ accumulate to produce the continuous spectrum of the limiting manifold. We prove that this is the case and obtain a sharp estimate of the rate of accumulation.
\end{abstract}

\section{INTRODUCTION}

According to the cusp closing theorem of Thurston [10], every complete noncompact three manifold $M_{0}$ of constant sectional curvature -1 and of finite volume is a limit of a sequence of compact hyperbolic manifolds $M_{k} \rightarrow M_{0}$. Since $M_{0}$ is not compact and its Laplacian has continuous spectrum [7], we expect the eigenvalues of $M_{k}$ to accumulate. A sharp estimate for the number of eigenvalues of the Laplacian on functions in any interval $\left[1,1+x^{2}\right]$ was given by Chavel and Dodziuk in [2]. For the Laplacian on forms of degree one the accumulation rate near zero was estimated by McGowan in [8].

In this paper we extend the results of [2] and [8] to the Laplacian acting on forms and an arbitrary interval $\left[0, x^{2}\right]$. Since we are dealing with three manifolds, the knowledge of the spectrum on functions and on forms of degree one determines the spectrum for forms of all degrees. Moreover, since the spectrum on functions is the same as the spectrum on exact forms of degree one (except for the multiplicity of zero as the eigenvalue), it will suffice to study the accumulation rate for the Laplacian restricted to coexact forms of degree one. We define $N_{k}(x)$ to be the number of the eigenvalues $\lambda$ of the Laplacian restricted to coexact forms of degree one on $M_{k}$ satisfying $\lambda \leq x^{2}$. Since the spectrum on coexact one forms is the same as the spectrum of the Laplacian restricted to exact two forms, $N_{k}(x)$ can be defined equivalently as the number of eigenvalues in the interval $\left[0, x^{2}\right]$ for the Laplacian on exact two forms. Various estimates will be carried out either for exact two forms or for coexact one forms depending on technical convenience.

Received by the editors May 24, 1994.

1991 Mathematics Subject Classification. Primary 58G25, 58A14.

The first author was supported in part by NSF Grant \#DMS-9204533 and the PSC-CUNY Research Award Program. 
If $M_{0}$ has $q$ cusps, then $M_{k}$ will contain $q$ simple, closed geodesics $\gamma_{k}^{i}, j=$ $1, \ldots, q$, of lengths $l\left(\gamma_{k}^{j}\right) \rightarrow 0$ and embedded tubular neighborhoods, referred to as tubes, of $\gamma_{k}^{j}$ of radius $R_{k}^{j} \rightarrow \infty$ as $k \rightarrow \infty$. The result of this paper is that as $k \rightarrow \infty$

$$
N_{k}(x)=2 \frac{x}{\pi} \sum_{j=1}^{q} R_{k}^{j}+O_{x}(1) .
$$

$O_{x}(1)$ stands for a bounded error whose magnitude depends on $x$.

The contents of the paper is as follows. In Section 2 we review the geometric setting, introduce some notation, and compute the Dirichlet integral $(d \omega, d \omega)+\left(d^{*} \omega, d^{*} \omega\right)$ for a differential form of degree one on a tube. Section 3 contains the proof of upper bounds for eigenvalues which yield a lower bound $N_{k}(x) \geq 2 \frac{x}{\pi} \sum_{j=1}^{q} R_{k}^{j}+O_{x}(1)$. The derivation of these bounds is reduced to proving bounds for eigenvalues of certain ordinary differential equations. The last three sections are devoted to lower bounds obtained using a general scheme originally due to Cheeger (unpublished) and later used in [8] by the second author. We review some consequences of the min-max principle in Section 4 and apply them to derive lower bounds for eigenvalues of the thick part of $M$ in Section 5. In the last section we reduce our problem to estimating eigenvalues of the same uncoupled ordinary differential equations that appeared in Section 3 and we complete the proof of (1.1).

Throughout most of the paper we will assume that $M_{0}$ has only one cusp so that $M_{k}$ contains only one short geodesic $\gamma=\gamma_{k}$ and, hence, only one tube $T=T_{\gamma}$ of radius $R_{k}$. This will be done only to simplify the notation and will not restrict the generality in any way. It will be clear from the proof that, in the case of more than one cusp, the contributions coming from various tubes are simply added. We will also omit the index $k$ and write $M, \gamma, R, N$ for $M_{k}, \gamma_{k}, R_{k}$, and $N_{k}$ respectively.

\section{ThE GEOMETRIC SETTING}

We consider compact hyperbolic manifolds of three dimensions, i.e. Riemannian three manifolds of constant sectional curvature -1 and their degeneration. A very readable survey of the geometry of such manifolds is contained in [5]. For a very thorough discussion of this topic see [10, Chapters 4, 5, 6].

We will use the following notation. For a Riemannian manifold $M$ and an interval $I, M_{I}=\{p \in M \mid l(p) \in I\}$, where $l(p)$ denotes the injectivity radius at $p \in M$. It is consequence of the Kazhdan-Margulis theorem [6], [10] that there exists a positive number $\mu$ such that, for every hyperbolic three manifold $M$, the set $M_{(\mu, \infty)}$ is nonempty and connected. $M_{(0, \mu]}$ consists of finitely many connected components which we will call tubes. They are metric tubular neighborhoods of simple closed geodesics in $M$ of length smaller than or equal to $2 \mu$. Let $\gamma$ be such a geodesic and let $\tilde{\gamma}$ be one of its lifts to the universal covering $\widetilde{M} \cong \mathbb{H}^{3}$. The corresponding tube $T=T_{\gamma}$ in $M$ is obtained as the quotient of a tubular neighborhood $\widetilde{T}$ of $\tilde{\gamma}$ by the cyclic group generated by $A=A_{\gamma}$, the deck transformation corresponding to $\gamma$. We use the Fermi coordinates $(r, t, \theta)$ in $\mathbb{H}^{3}$ based on $\tilde{\gamma} . \quad r$ denotes the distance from $\tilde{\gamma}, t$ is the arclength along $\tilde{\gamma}$ and $\theta$ is the angular coordinate in the circle of unit 
vectors perpendicular to $\tilde{\gamma}$ at a point. To make a consistent choice of $\theta$ we choose and fix a parallel field of unit vectors perpendicular to $\tilde{\gamma}$. In terms of these coordinates the metric of $\mathbb{H}^{3}$ is expressed as

$$
d s^{2}=d r^{2}+\cosh ^{2} r d t^{2}+\sinh ^{2} r d \theta^{2},
$$

and the deck transformation $A$ is given by $A(r, t, \theta)=(r, t+l(\gamma), \theta+\alpha)$ for some angle $\alpha . \widetilde{T}=\{\tilde{p} \in \widetilde{M} \mid d(\tilde{p}, \tilde{\gamma}) \leq R\}$, and $T=\widetilde{T} /\left\langle A_{\gamma}\right\rangle$ is determined up to isometry by $R, \alpha$, and $l=l(\gamma)$. It is proved in Section 2 of [2] that there exist universal constants $c_{1}, c_{2}>0$ such that

$$
c_{1} \leq l \sinh ^{2} R \leq c_{2} \operatorname{vol}\left(M_{0}\right) \text {. }
$$

In particular, if the volume is bounded and $l$ approaches zero, $R$ tends to infinity, $R \sim \frac{1}{2} \log \left(\frac{1}{l}\right)$.

Recall that we are interested in a noncompact hyperbolic manifold $M_{0}$ of finite volume and a sequence of compact hyperbolic manifolds $\left(M_{k}\right)_{k=1}^{\infty}$ converging to $M_{0}[5, \S 3]$. It is known [10] that $\operatorname{vol}\left(M_{k}\right) \leq \operatorname{vol}\left(M_{0}\right)$.

If $M_{0}$ has $q$ cusps, we can find a positive number $\varepsilon \leq \mu$ and a sequence $\varepsilon_{k} \searrow 0$ such that each $M_{k}$ contains exactly $q$ geodesics $\gamma_{k}^{1}, \gamma_{k}^{2}, \ldots, \gamma_{k}^{q}$ of length less than or equal to $\varepsilon_{k}$ and that for every $k$ the injectivity radius at every point of $M_{i} \backslash \bigcup_{j=1}^{q} T_{\gamma_{k}^{j}}$ is greater than or equal to $\varepsilon$. Let $l\left(\gamma_{k}^{j}\right)=l_{k}^{j}$ and let $R_{k}^{j}$ be the radius of $T_{\gamma_{k}^{j}}$. As above,

$$
R_{k}^{j}=\frac{1}{2} \log \left(\frac{1}{l_{k}^{j}}\right)+O\left(\log \operatorname{vol}\left(M_{0}\right)\right) .
$$

Thus $M_{k}$ contains exactly $q$ tubes of radii tending to infinity when $k \rightarrow \infty$. These tubes become cusps in the limit. The boundary of a tube with the induced metric is a flat torus. The tori $F_{k}^{j}=\partial T_{\gamma_{k}^{j}}$ are nondegenerate in the following sense (cf. [2, Section 2] for the proof).

Lemma 2.2. There exists a universal constant $\kappa>0$ such that $F_{k}^{j}$ with its induced metric has injectivity radius at every point greater than or equal to $\kappa$. Moreover $\operatorname{vol}\left(F_{k}^{j}\right) \leq c \operatorname{vol}(M)$ for a constant $c$ independent of $k$. Therefore the tori $F_{k}^{j}$ form a relatively compact family.

We conclude this section by introducing a moving frame of one forms on the tube $T=T_{\gamma}$ and using it for a computation of the Dirichlet integral. Consider the forms $d r, d t, d \theta$ on $\widetilde{M}$ introduced above. It is clear that they descend to well-defined forms on $T$. We denote the dual vector fields by $\frac{\partial}{\partial r}, \frac{\partial}{\partial t}, \frac{\partial}{\partial \theta}$ respectively. Let

$$
\omega=f d r+\alpha,
$$

where $f$ is a function and $\alpha=g_{1} d t+g_{2} d \theta$ a one form which does not contain $d r$. We need to compute $(d \omega, d \omega)+\left(d^{*} \omega, d^{*} \omega\right)$, where $d^{*}$ is the formal adjoint of the exterior derivative and $(\alpha, \beta)=\int_{M} \alpha \wedge * \beta$ is the $L^{2}$ inner product of forms $\alpha$ and $\beta$. To do so we introduce the following notation. Let $F_{r}=\{x \in T \mid d(x, \gamma)=r\}$ be the torus at distance $r$ from $\gamma$. It is flat with respect to the induced metric. We denoted $d^{r}, *^{r}, \Delta^{r}$ the exterior derivative operator, the Hodge star operator and the Laplacian on $F_{r}$ with its induced 
metric. In addition, set $d^{*}=\vartheta$ and let $\vartheta^{r}$ be the adjoint of the exterior derivative $d^{r}$ of $F_{r}$. A straightforward calculation shows that

$$
\begin{aligned}
d \omega & =d^{r} f \wedge d r+d r \wedge \frac{\partial \alpha}{\partial r}+d^{r} \alpha \\
* \omega & =f \sinh r \cosh r d t \wedge d \theta+*^{r} \alpha \wedge d r \\
d * \omega & =\frac{\partial}{\partial r}(f \sinh r \cosh r) d r \wedge d t \wedge d \theta+d^{r} *^{r} \alpha \wedge d r
\end{aligned}
$$

Introduce the differential operator

$$
L f=\frac{1}{\sinh 2 r} \frac{\partial}{\partial r}(\sinh 2 r f) \text {. }
$$

Clearly, $L$ is the formal adjoint of $-\partial / \partial r$ with respect to the inner product in $L^{2}(\sinh r \cosh r d r)$. Using the formulae above and the fact that $\vartheta^{r} \beta=$ $-*^{r} d^{r} *^{r} \beta$ for a form of degree one on $F_{r}$ we obtain the following expression for the Dirichlet integral.

$$
\begin{gathered}
(d \omega, d \omega)+(\vartheta \omega, \vartheta \omega)=(L f, L f)+\left(\frac{\partial \alpha}{\partial r}, \frac{\partial \alpha}{\partial r}\right)+\left(d^{r} f, d^{r} f\right) \\
+\left(d^{r} \alpha, d^{r} \alpha\right)+\left(\vartheta^{r} \alpha, \vartheta^{r} \alpha\right)-2\left(d^{r} f, \frac{\partial \alpha}{\partial r}\right)-2\left(L f, \vartheta^{r} \alpha\right) .
\end{gathered}
$$

We remark that in an application of this formula the last two terms on the second line will cancel, since, for forms satisfying appropriate boundary conditions, the boundary terms in integration by parts with respect to $r$ will vanish.

\section{UPPER BOUNDS}

Let $0<\nu_{1} \leq \nu_{2} \leq \cdots \rightarrow \infty$ be the sequence of eigenvalues of the Laplacian on $M=M_{k}$ restricted to coexact forms of degree one. We wish to derive upper bounds for $\nu_{j}, j=1,2, \ldots$. Consider the space $\mathscr{E}$ of $C^{\infty}$ forms of degree one on $M$ with supports contained in the set $\{x \in T \mid 1 \leq d(x, \gamma) \leq R\}$ whose coefficients in terms of the orthonormal frame $d r, d t, d \theta$ depend only on $r$, and which are coexact. By Courant's min-max principle the eigenvalues $\nu_{j}$ are smaller than or equal to the critical values of the Rayleigh-Ritz quotient $(\Delta \omega, \omega) /(\omega, \omega)$ restricted to $\mathscr{E}$. Every form $\omega \in \mathscr{E}$ can be written as $\omega=$ $f(r) d r+g_{1}(r) d t+g_{2}(r) d \theta$. Since $\omega$ is assumed to be coexact, its inner product with the exterior derivative of an arbitrary function $u$ has to vanish. Taking the inner product with $d u$ and integrating by parts we see that $f(r)$ has to be constant and hence equal to zero. $g_{1}$ and $g_{2}$ vanish outside the interval $[1, R]$ and satisfy

$$
\int_{1}^{R} g_{1}(r) \tanh r d r=0, \quad \int_{1}^{R} g_{2}(r) \operatorname{coth} r d r=0
$$

since $g_{1}(r) \tanh r$ and $g_{2}(r) \operatorname{coth} r$ are $r$-derivatives by coexactness.

Thus for a form $\omega \in \mathscr{E}$ we have $(\Delta \omega, \omega)=(d \omega, d \omega)$ and

$$
\begin{gathered}
(d \omega, d \omega)=2 \pi l(\gamma) \int_{1}^{R}\left(g_{1}^{\prime}\right)^{2} \tanh r d r+2 \pi l(\gamma) \int_{1}^{R}\left(g_{2}^{\prime}\right)^{2} \operatorname{coth} r d r, \\
(\omega, \omega)=2 \pi l(\gamma) \int_{1}^{R} g_{1}^{2} \tanh r d r+2 \pi l(\gamma) \int_{1}^{R} g_{2}^{2} \operatorname{coth} r d r .
\end{gathered}
$$


Therefore the critical values of $(\Delta \omega, \omega)$ on $\mathscr{E}$ are the union of sets of critical values of the two Rayleigh-Ritz quotients defined below.

$$
\begin{aligned}
& \mathscr{R}_{1}\left(g_{1}\right)=\frac{\int_{1}^{R}\left(g_{1}^{\prime}\right)^{2} \tanh r d r}{\int_{1}^{R} g_{1}^{2} \tanh r d r}, \\
& \mathscr{R}_{2}\left(g_{1}\right)=\frac{\int_{1}^{R}\left(g_{2}^{\prime}\right)^{2} \operatorname{coth} r d r}{\int_{1}^{R} g_{1}^{2} \operatorname{coth} r d r},
\end{aligned}
$$

where the prime denotes differentiation with respect to $r$.

Integrating by parts in the numerator of $\mathscr{R}_{1}$ we see that the critical values of $\mathscr{R}_{1}\left(g_{1}\right)$ are the eigenvalues of the Sturm-Liouville problem

$$
-\frac{1}{\tanh r}\left(g_{1}^{\prime} \tanh r\right)^{\prime}=\mu g_{1}, \quad g_{1}(1)=g_{1}(R)=0 .
$$

The substitution $g_{1}(r)=u(r) \tanh ^{-1 / 2} r$ transforms this into

$$
-u^{\prime \prime}-\frac{1+4 \sinh ^{2} r}{4 \sinh ^{2} r \cosh ^{2} r} u=\mu u, \quad u(1)=u(R)=0 .
$$

The potential $\left(1+4 \sinh ^{2} r\right)\left(4 \sinh ^{2} r \cosh ^{2} r\right)^{-1}$ is integrable at infinity. By [2, Theorem 4.1], the number $K_{1}(x)$ of eigenvalues of this problem below the level $x^{2}$ satisfies $K_{1}(x)=\frac{x(R-1)}{\pi}+O_{x}(1)=\frac{x R}{\pi}+O_{x}(1)$. Similar analysis for $\mathscr{R}_{2}(x)$ yields $K_{2}(x)=\frac{x R}{\pi}+O_{x}(1)$, where $K_{2}(x)$ is the number of critical values of $\mathscr{R}_{2}$ below the level $x^{2}$. Thus the total number of critical values $\mu_{j}$ of $\mathscr{R}_{1}$ and $\mathscr{R}_{2}$ smaller than $x^{2}$ is equal to $\frac{2 x R}{\pi}+O_{x}(1)$. Since $\nu_{j} \leq \mu_{j}$ for all $j$, we see that

$$
N(x) \geq \frac{2 x R}{\pi}+O_{x}(1)
$$

\section{HODGE THEORY AND MIN-MAX PRINCIPLE}

We will have to consider manifolds with mildly singular boundaries. The worst case will be an intersection of a number of convex sets with smooth boundaries in $\mathbb{H}^{3}$. The Riemannian metric in every case will be the restriction of a $C^{\infty}$ metric on the ambient manifold. For such a manifold $\bar{M}$ we define the Laplacian $\Delta=\Delta_{N}$ on forms of degree $p$ as an unbounded operator on the space $L^{2} A^{p}(M)$ of square integrable forms of degree $p$ on $M=\bar{M} \backslash \partial M$. Let $A^{p}(M)$ denote the space of smooth forms of degree $p$ on $M$ and let $d$ and $\vartheta_{c}$ be the exterior derivative and its formal adjoint with domains

$$
\begin{aligned}
\operatorname{dom}(d) & =\left\{\omega \in A^{p}(M) \mid d \omega \in L^{2} A^{p}(M)\right\}, \\
\operatorname{dom}\left(\vartheta_{c}\right) & =\left\{\omega \in A^{p}(M) \mid \operatorname{supp}(\omega) \text { is compact }\right\} .
\end{aligned}
$$

Let $\bar{d}$ and $\overline{\vartheta_{c}}$ be the $L^{2}$ closures of $d$ and $\vartheta_{c}$ respectively and define

$$
\Delta_{N}=\bar{d} \circ \overline{\vartheta_{c}}+\overline{\vartheta_{c}} \circ \bar{d}
$$

with the usual conventions about domains. If $M$ has smooth boundary and $\omega$ is smooth on $\bar{M}$, then $\omega \in \operatorname{dom}\left(\Delta_{N}\right)$ if and only if $\omega$ satisfies the absolute boundary conditions

$$
\omega_{\text {norm }}=0, \quad d \omega_{\text {norm }}=0
$$


on $\partial M$. Therefore we will say that $\omega$ satisfies the absolute boundary conditions if $\omega \in \operatorname{dom}\left(\Delta_{N}\right)$. To simplify the notation we will write $\Delta, d, \vartheta$ for $\Delta_{N}, \operatorname{vol} \bar{d}, \overline{\vartheta_{c}}$ respectively. It will also be assumed implicitly that a form to which an operator is applied is in the domain of this operator. For example, if we say that a form $\omega$ is an eigenform of $\Delta$, we mean that $\omega$ is an eigenform and satisfies the absolute boundary conditions.

For manifolds under consideration (cf. [3], [4]) the Laplacian $\Delta$ is selfadjoint and has pure point spectrum. Moreover, all familiar results of Hodge theory hold. In particular, if $\lambda>0$ is an eigenvalue of $\Delta$ and $\phi \in A^{p}(M)$ is the corresponding eigenform, then

$$
\phi=\frac{1}{\lambda}(d \vartheta \phi+\vartheta d \phi)
$$

where the two summands are orthogonal in $L^{2} A^{p}(M)$ and are themselves eigenforms with eigenvalue $\lambda$. Thus the $\lambda$-eigenspace $E^{p}(\lambda)$ splits into orthogonal direct sum

$$
E^{p}(\lambda)=E_{d}^{p}(\lambda) \oplus E_{\vartheta}^{p}(\lambda)
$$

where $E_{d}^{p}(\lambda)$ and $E_{\theta}^{p}(\lambda)$ denote spaces of all exact and coexact eigenforms belonging to the eigenvalue $\lambda$ respectively.

For $\lambda>0$ define $T: E_{d}^{p}(\lambda) \rightarrow E_{\vartheta}^{p-1}(\lambda)$ by

$$
T \phi=\frac{1}{\lambda} \vartheta \phi
$$

It is very easy to check that $d \circ T \phi=\phi, T \circ d \psi=\psi$ and that $(T \phi, T \phi)=$ $\lambda^{-1}(\phi, \phi)$. In particular, $T$ extends to a bounded operator defined on the $L^{2}$ closure of the space of exact $p$-forms and $T \phi=\psi$ is the unique coexact form on $M$ satisfying $d \psi=\phi$

Let $\phi_{1}, \phi_{2}, \ldots$ be a complete orthonormal set of exact eigenforms of degree $p$ and let $0<\lambda_{1} \leq \lambda_{2} \leq \cdots$ be corresponding eigenvalues. Clearly, $\psi_{i}=$ $\lambda_{i}^{1 / 2} T \phi_{i}$ is a complete orthonormal set in the space of coexact forms of degree $p-1$. Set $\mathscr{F}_{i}=\operatorname{span}\left\{\phi_{1}, \ldots, \phi_{i}\right\}$. Our lower bounds for eigenvalues will follow from the following simple consequences of the min-max principle.

Lemma 4.2. The eigenvalues $\lambda_{i}$ are given by

$$
\sup \left\{\frac{(\phi, \phi)}{(\eta, \eta)} \mid \phi \in \mathscr{F}_{i} \backslash\{0\}, \eta \in A^{p-1}(M), d \eta=\phi\right\} .
$$

Proof. Let $\psi=T \phi$ for $\phi=\sum a_{j} \phi_{j}$. Then $\phi=d \psi$ and

$$
\frac{(\phi, \phi)}{(\psi, \psi)}=\frac{\sum a_{j}^{2}}{\sum \lambda_{j}^{-1} a_{j}^{2}} .
$$

Since $\lambda_{i}=\max _{j} \lambda_{j}$, we see that

$$
\lambda_{i}=\sup \left\{\frac{(\phi, \phi)}{(\psi, \psi)} \mid \phi \in \mathscr{F}_{i} \backslash\{0\}\right\} .
$$

Now observe that if $\phi=d \eta$, then $\eta=\psi+\alpha$ for some closed form $\alpha$. This follows from the Hodge decomposition. Thus, for a fixed $\phi$,

$$
\frac{(\phi, \phi)}{(\psi, \psi)}=\sup \left\{\frac{(\phi, \phi)}{(\eta, \eta)} \mid d \eta=\phi\right\} .
$$

This finishes the proof. 
The following is a useful reformation of the lemma. If $\phi \in \mathscr{F}_{i} \backslash\{0\}, d \eta=\phi$ and $(\phi, \phi) \geq c(\eta, \eta)$, then $\lambda_{i} \geq c$.

Another simple observation will be used repeatedly in the sequel $\left(\mathscr{F}_{0}\right.$ is empty by convention).

Lemma 4.3. Suppose $\phi$ is an exact form perpendicular to $\mathscr{F}_{m}$. Then

$$
(\phi, \phi) \geq \lambda_{m+1}(\psi, \psi)
$$

where $\psi=T \phi$.

Proof. By assumption $\phi=\sum_{i \geq m+1} a_{i} \phi_{i}$. Therefore, since $T \phi_{i}=\lambda_{i}^{-1} \vartheta \phi_{i}$,

$$
(\psi, \psi)=\sum_{i \geq m+1} \lambda_{i}^{-1} a_{i}^{2} \leq \lambda_{m+1}^{-1}(\phi, \phi) .
$$

Our scheme for proving lower bounds will be as follows. We will start with $\phi \in \mathscr{F}_{i}$ and will attempt to construct a form $\psi$ with $d \psi=\phi$ in such a way that we can estimate $(\psi, \psi)$ in terms of $(\phi, \phi)$. In order to carry out the construction and to be able to obtain good estimates we will have to impose certain linear conditions on $\phi$. If $i=\operatorname{dim} \mathscr{F}_{i}$ is greater than the number $C$ of conditions imposed, then $\mathscr{F}_{i}$ will contain a nonzero form satisfying these conditions. Thus, using Lemma 4.2 applied with $i=C+1$, we obtain a lower bound for $\lambda_{C+1}$.

\section{THE THICK PART OF $M$}

From now on $M$ is as in the introduction; i.e. $M=M_{k}$ for some positive integer $k$, where the compact hyperbolic manifolds $M_{k}$ converge to a finite volume manifold of sectional curvature identically equal to -1 with one cusp. We use the method of [8] to estimate the number of eigenvalues of the thick part of $M$. Recall that there exists a number $\varepsilon>0$ such that the injectivity radius at every point of the complement of the tube is greater than or equal to $\varepsilon$; i.e. $M \backslash T=M_{(\varepsilon, \infty)}$. For technical reasons we consider $M^{\prime}=$ $M \backslash\left\{p \in T \mid d(p, \partial T)>r_{0}\right\}$ instead of $M_{[\varepsilon, \infty)}$. We recall from Section 4 that every eigenform on a manifold with boundary, in particular on $M^{\prime}$, is assumed to satisfy the boundary conditions (4.1).

The main result of this section is the following proposition.

Proposition 5.1. For fixed $y>0$ and $r_{0}>5 \varepsilon$, the number of eigenvalues $\nu_{j}$ on exact forms on $M^{\prime}$ of degree two satisfying $\nu_{j} \leq y^{2}$ is bounded by a constant $a=a\left(y, r_{0}\right)$. The constant is independent of $k$, i.e. does not depend on where $M$ occurs in the sequence $\left(M_{k}\right)_{k=1}^{\infty}$.

Proof. We use the Čech-de Rham formalism as in [1, Chapter 2] and [8]. In particular, we follow the notation of [1]. First, we have to construct a covering of $M^{\prime}$ by open sets of known geometry. Choose a small positive number $\alpha<\varepsilon / 3$. We treat $\alpha$ as a parameter whose value will be specified later. In addition, we adopt the convention that all constants $c_{k}$, with $k$ a positive integer, are universal, i.e. do not depend on $\alpha, r_{0}$ or $M$.

Choose a maximal set $\mathscr{P}$ of points in $M_{[\varepsilon, \infty)}$ with pairwise distances greater than or equal to $\alpha$. The balls $B(p, \alpha / 2), p \in \mathscr{P}$, are disjoint and the balls 
$B(p, \alpha)$ cover $M_{[\varepsilon, \infty)}$. We will work with the covering $\mathscr{U}$ of $M^{\prime}$ consisting of all balls $B(p, 3 \alpha), p \in \mathscr{P}$, and the set $S=\left\{p \in T \mid d(p, \partial T)<r_{0}\right\}$. Write $\mathscr{U}=\left\{U_{i}\right\}_{i \in I}$, where $I$ is a finite index set. The cardinality of $I$ can be estimated in terms of $\alpha$ only since $\operatorname{vol}(M)<\operatorname{vol}\left(M_{0}\right)$ and the balls of radius $\alpha / 2$ centered at $p \in \mathscr{P}$ are disjoint. In addition, the degree of overlap $\max _{p \in M^{\prime}} \#\left\{i \in I \mid p \in U_{i}\right\}$ has a universal bound independent of $\alpha$. We will also need a partition of unity $\left\{\rho_{i}\right\}$ subordinate to this covering with the property that

$$
\left\|d \rho_{i}\right\|_{\infty} \leq \frac{c_{1}}{\alpha}
$$

Such a partition of unity can be constructed as follows. If $U_{i}=B\left(p_{i}, \alpha\right)$ is a ball, define the function $\overline{\rho_{i}}$ as follows.

$$
\overline{\rho_{i}}(p)= \begin{cases}1 & \text { if } p \in B\left(p_{i}, 2 \alpha\right) \\ 1-\frac{d\left(p, B\left(p_{i}, 2 \alpha\right)\right)}{\alpha} & \text { if } p \in B\left(p_{i}, 3 \alpha\right) \backslash B\left(p_{i}, 2 \alpha\right) \\ 0 & \text { otherwise. }\end{cases}
$$

If $U_{i}=S$, then

$$
\overline{\rho_{i}}(p)= \begin{cases}1 & \text { if } p \in S \text { and } d(p, \partial T) \geq \alpha ; \\ \frac{d(p, \partial T)}{\alpha} & \text { if } p \in S \text { and } d(p, \partial T) \leq \alpha ; \\ 0 & \text { on } M^{\prime} \backslash S .\end{cases}
$$

It is clear that the supports of $\rho_{i}$ form a locally finite family and $\rho_{i}=\overline{\rho_{i}} / \sum_{i} \overline{\rho_{i}}$ have the required properties.

In the course of the argument we will need information about eigenvalues for the Laplacian on exact forms of degree two on the sets $U_{i}$, and eigenvalues for exact forms of degree one on the intersections $U_{i, j}=U_{i} \cap U_{j}$. We first note that if $U_{i}$ is a ball of radius $3 \alpha$ in our covering, then the smallest eigenvalue $\mu$ of the Laplacian on exact forms of degree two satisfies

$$
\mu \geq \frac{c_{2}}{\alpha^{2}}
$$

This can be seen, for example, by replacing the metric on $B(p, 3 \alpha)$ by the Euclidean metric on a Euclidean ball of equal radius (cf. [8, Lemma 2.2]) for which the estimate follows by scaling. On the other hand, the shell $S$ is quasiisometric (with constants controlling quasi-isometry depending only on $r_{0}$ ) to $T \times\left[0, r_{0}\right]$. This is a consequence of $(2.1)$ and of Lemma 2.2. Thus the number of eigenvalues of $S$ below the level $z^{2}$ can be bounded by a constant depending only on $z$ and $r_{0}$ (cf. [8, Lemma 2.2]).

Next consider double intersections $U_{i, j}$. The eigenvalues on exact forms of degree one are the same as the positive eigenvalues on functions satisfying Neumann boundary conditions. Note that all $U_{i, j}$ are convex in the hyperbolic metric. Working in the upper half-space model of the hyperbolic space and replacing the hyperbolic metric by the flat metric associated to standard coordinates, we can assume that $U_{i, j}$ are convex with respect to the Euclidean metric and that the two metrics are quasi-isometric (with constants independent of $r_{0}, \alpha$ and $M$ ) (cf. Section 4.1 of [8]). It then follows from [9] that the smallest positive eigenvalue $\mu$ of the Laplacian on $U_{i, j}$ satisfies

$$
\mu \geq \frac{c_{3}}{\alpha^{2}} \text {. }
$$


After these preliminaries we continue the proof. Take $\phi \in \mathscr{F}_{m}$. Since $\phi$ is exact on $M$, its restriction $\phi_{i}$ to every $U_{i}$ is exact and there exists a unique coexact form $\psi_{i}$ on $U_{i}$ satisfying $d \psi_{i}=\phi_{i}$. If $U_{i}$ is a ball, we have, by Lemma 4.3 and (5.3),

$$
\left(\phi_{i}, \phi_{i}\right)_{U_{i}} \geq \frac{c_{2}}{\alpha^{2}}\left(\psi_{i}, \psi_{i}\right)_{U_{i}},
$$

where $(\omega, \eta)_{X}$ denotes the inner product of forms on a manifold $X$.

In order to have the same inequality for $U_{i}=S$ we have to impose some conditions on $\phi$. Namely, we have to require that $\phi \mid U_{i}=\phi_{i}$ be such that $\psi_{i}$ is orthogonal to all coexact eigenforms of $S$ with eigenvalues smaller than $c_{2} / \alpha^{2}$. As remarked above, the number of such eigenvalues of $S$ is bounded by a constant that depends only on $\alpha$ and $r_{0}$. In other words, after imposing finitely many conditions, we can assume that (5.5) holds for every $i$.

The forms $\psi_{i}$ do not define a global form on $M^{\prime}$. Consider $\left\{\psi_{i}\right\}$ as a Čech cochain. The obstruction to $\psi_{i}$ 's being restrictions of globally defined form is the coboundary $\delta\left\{\psi_{i}\right\}=\left\{\omega_{i, j}\right\}$, where $\omega_{i, j}=\psi_{j}-\psi_{i}$ on $U_{i, j}$. Clearly, $d \omega_{i, j}=d \psi_{j}-d \psi_{i}=\phi_{j}-\phi_{i}=0$ on $U_{i, j}$. Since all $U_{i, j}$ are convex, $\omega_{i, j}=d g_{i, j}$ for a unique function $g_{i, j}$ perpendicular to constants in $L^{2}\left(U_{i, j}\right)$. In view of $(5.4)$

$$
\left(\omega_{i, j}, \omega_{i, j}\right)_{U_{i, j}} \geq \frac{c_{3}}{\alpha^{2}}\left(g_{i, j}, g_{i, j}\right)_{U_{i, j}} .
$$

The Čech coboundary $\delta\left\{g_{i, j}\right\}=\left\{h_{i, j, k}\right\}=\left\{g_{j, k}-g_{i, k}+g_{i, j}\right\}$ is not necessarily equal to zero. However, $d\left\{h_{i, j, k}\right\}=d \delta\left\{g_{i, j}\right\}=\delta\left\{d g_{i, j}\right\}=\delta\left\{\omega_{i, j}\right\}=$ $\delta \delta\left\{\psi_{i}\right\}=0$. Therefore, since all triple intersections $U_{i, j, k}$ are convex, $h_{i, j, k}$ are constant. Note that these constants are uniquely determined by the original form $\phi$. We impose yet another set of conditions (equal in number to the number of nonempty triple intersections) requiring that $h_{i, j, k}$ be zero for all $i, j, k$ such that $U_{i} \cap U_{j} \cap U_{k} \neq \varnothing$. The total number of conditions imposed so far is finite and is determined by $\alpha$ and $r_{0}$ only. We now use formula (8.6) of [1] to modify $\psi_{i}$. Define $\tau_{i}=\sum_{k} \rho_{k} g_{k, i}$ and $\eta_{i}=\psi-d \tau_{i}$. The coboundary $\delta\left\{\tau_{i}\right\}=\left\{g_{i, j}\right\}$ (cf. [1, Proposition 8.5]) so that

$$
\delta\left\{\eta_{i}\right\}=\delta\left\{\psi_{i}\right\}-\delta d\left\{\tau_{i}\right\}=\left\{\omega_{i, j}\right\}-\left\{d g_{i, j}\right\}=0 .
$$

This is equivalent to saying that the family $\left\{\eta_{i}\right\}$ consists of restrictions of a globally defined form $\eta$ such that $d \eta=\phi$.

It remains to estimate the $L^{2}$ norm of $\eta$. Clearly,

$$
(\eta, \eta)_{M^{\prime}} \leq \sum_{i}\left(\eta_{i}, \eta_{i}\right)_{U_{i}}
$$

and, for a fixed $i$,

$$
\eta_{i}=\psi_{i}+\sum_{k} d \rho_{i} g_{k, i}+\sum_{k} \rho_{k} \omega_{k, i}
$$

Both summations above extend over the set of those indices $k$ for which $U_{i} \cap$ $U_{k} \neq 0$. We observed above that the number of such indices can be estimated from above independently of $\alpha$ and $r_{0}$. This observation combined with (5.2) 
and (5.6) yields

$$
\left\|\sum_{k} d \rho_{k} g_{k, i}\right\|_{U_{i}}^{2} \leq \frac{c_{1}}{\alpha^{2}} \sum_{k}\left\|g_{k, i}\right\|_{U_{k, i}}^{2} \leq c_{4} \sum_{k}\left\|\omega_{k, i}\right\|_{U_{k, i}}^{2} .
$$

In addition,

$$
\left\|\sum_{k} \rho_{k} \omega_{k, i}\right\|_{U_{i}}^{2} \leq c_{5} \sum_{k}\left\|\omega_{k, i}\right\|_{U_{k, i}}^{2} .
$$

On the other hand, $\omega_{i, j}=\psi_{j}-\psi_{i}$ on $U_{i, j}$ so that

$$
\left\|\omega_{k, i}\right\|_{U_{k, i}}^{2} \leq 2\left(\left\|\psi_{i}\right\|_{U_{i}}^{2}+\left\|\psi_{k}\right\|_{U_{k}}^{2}\right) \text {. }
$$

(5.5), (5.7), (5.8) and (5.9) together yield the following estimate.

$$
\frac{c_{6}}{\alpha^{2}}\|\eta\|^{2} \leq\|\phi\|^{2} \text {. }
$$

At this point we choose the value for $\alpha$ so that $\frac{c_{6}}{\alpha^{2}} \geq y^{2}$, where $y$ is as in the statement of the proposition.

The estimate above holds provided the form $\phi$ satisfies all the conditions imposed in the course of the argument. The number $a=a\left(y, r_{0}\right)$ of these conditions is finite and after taking $m=a+1$ we can find a nonzero form in $\phi \in \mathscr{I}_{m}$ which satisfies all the conditions. The proposition now follows from Lemma 4.2.

\section{ANALYSIS ON THE TUBE}

In this section we do analysis on the tube to complete the proof of (1.1). Consider the covering of $M$ consisting of two open sets $U_{1}=M^{\prime} \backslash \partial M^{\prime}$ and $U_{2}=\left\{x \in T \mid d(x, \partial T)>r_{0}-1\right\}$.

Take an exact form $\phi$ on $M$ whose restriction $\phi_{1}$ to $U_{1}$ is orthogonal to finite-dimensional space spanned by exact eigenforms of degree two on $M^{\prime}$ with eigenvalues less than or equal to $y^{2}$. The values of $y$ and $r_{0}$ will be specified below. We will say that a form $\omega$ on the tube has coefficients that depend only on the $r$ variable, $r=d(x, \gamma)$, if and only if the coefficients with respect to the frame $d r, d t, d \theta$ depend only on $r$. For every $r, 0 \leq r \leq R$, we define $F_{r}$ to be the flat torus at distance $r$ from $\gamma$. Every differential form $\omega$ on a subset $\left\{x \in T \mid r_{1}<d(x, \gamma)<r_{2}\right\}$ of $T$ can be decomposed as follows.

$$
\omega=\bar{\omega}+\overline{\bar{\omega}}
$$

where coefficients of $\bar{\omega}$ depend only on $r$ and the coefficients of $\overline{\bar{\omega}}$ are perpendicular in $L^{2}$ to constants on every distance torus $F_{r} . \bar{\omega}$ is computed by averaging the coefficients of $\omega$ on each torus $F_{r}$. It follows that the operation $\omega \mapsto \bar{\omega}$, and therefore $\omega \mapsto \overline{\bar{\omega}}$, commute with the Hodge star operator, exterior derivative, its adjoint and the Laplacian. In addition,

$$
(\bar{\omega}, \overline{\bar{\omega}})_{F_{r}}=0
$$

for all $r$. 
By assumption and Lemma 4.3 there exists a unique coexact form of degree one $\psi_{1}$ on $U_{1}$ such that

$$
d \psi_{1}=\phi_{1}=\phi \mid U_{1} \text { and } y^{2}\left(\psi_{1}, \psi_{1}\right)_{U_{1}} \leq\left(\phi_{1}, \phi_{1}\right)_{U_{1}} .
$$

Similarly, there exists a unique coexact form $\psi_{2}$ such that $d \psi_{2}=\phi_{2}=d \phi \mid U_{2}$. We will require certain additional properties of $\psi_{1}$ and $\psi_{2}$ which will amount to more conditions on $\phi$. Decompose $\phi_{i}, \psi_{i}, i=1,2$, as in (6.1). We will use the method of Section 5 to splice $\overline{\overline{\psi_{1}}}$ with $\overline{\overline{\psi_{2}}}$, but we have to be more careful when treating $\bar{\phi}$.

Consider $\overline{\overline{\psi_{1}}}$ and $\overline{\overline{\psi_{2}}}$ first. Let $h(d)$ be the function defined as follows.

$$
h(d)= \begin{cases}0 & \text { if } d \leq \frac{1}{4} \\ 2\left(d-\frac{1}{4}\right) & \text { if } \frac{1}{4} \leq x \leq \frac{3}{4} \\ 1 & \text { if } d \geq \frac{3}{4}\end{cases}
$$

and $\rho(p)=h\left(d(p, \partial T)-r_{0}+1\right) . \overline{\overline{\psi_{1}}}-\overline{\overline{\psi_{2}}}$ is closed on $U_{1} \cap U_{2}$ and we require it to be exact. Since $U_{1} \cap U_{2}$ retracts to a torus, this amounts to two additional linear conditions on $\phi$. Thus $\overline{\overline{\psi_{1}}}-\overline{\overline{\psi_{2}}}=d g$ and we can assume without loss of generality that $g=\overline{\bar{g}}$ is a function perpendicular to constants on every torus $F_{r} \subset U_{1} \cap U_{2}$. We then define

$$
\eta_{1}=\overline{\overline{\psi_{1}}}-d(\rho g)
$$

on $U_{1} \cap T$ and

$$
\eta_{2}=\overline{\overline{\psi_{2}}}-d((1-\rho) g)
$$

on $U_{2}$. One checks that $\eta_{1}=\eta_{2}$ on $U_{1} \cap U_{2}$ so that we obtain a form $\eta$ on $T$ such that $\eta \mid U_{i} \cap T=\eta_{i}$. As above we need to estimate the $L^{2}$ norm of $\eta$. To do this we use the following lemma.

Lemma 6.3. We have the following inequalities.

$$
\left\|\overline{\overline{\psi_{1}}}-\overline{\overline{\psi_{2}}}\right\|_{U_{1} \cap U_{2}}^{2} \geq \nu\left(R-r_{0}+1\right)\|g\|_{U_{1} \cap U_{2}}^{2}
$$

and

$$
\left\|\overline{\overline{\phi_{2}}}\right\|_{U_{2}}^{2} \geq \nu\left(R-r_{0}+1\right)\left\|\overline{\overline{\psi_{2}}}\right\|_{U_{2}}^{2}
$$

where $\nu(r)$ is the first positive eigenvalue of the Laplacian on functions on the torus $F_{r}$.

Note that the behavior of the function $\nu(r)$ is described in Lemma 3.9 of [2]. $\nu(r)$ is decreasing and

$$
\nu(r) \geq c_{7} e^{2(R-r)} .
$$

In the argument below we will fix the value of $r_{0}$ so that $\nu\left(R-r_{0}+1\right) \geq$ $c_{7} e^{2\left(r_{0}-1\right)} \geq y^{2}$. We defer the proof of the lemma and proceed with the estimate. On $T \backslash U_{2}, \eta=\overline{\overline{\psi_{1}}}$ and we have good control over the norm of $\psi_{1}$, and hence of $\overline{\overline{\psi_{1}}}$, by assumption. On the overlap $U_{1} \cap U_{2}$

$$
\eta=\eta_{2}=\overline{\overline{\psi_{2}}}+g d \rho-(1-\rho)\left(\overline{\overline{\psi_{1}}}-\overline{\overline{\psi_{2}}}\right),
$$

and

$$
\eta=\overline{\overline{\psi_{2}}}
$$


on $U_{2} \backslash U_{1}$. Thus

$$
\|\eta\|_{U_{1} \cap U_{2}}^{2} \leq c_{8}\left(\left\|\overline{\overline{\psi_{2}}}\right\|_{U_{2}}^{2}+\left\|\overline{\overline{\psi_{1}}}\right\|_{U_{1}}^{2}+\|g\|_{U_{1} \cap U_{2}}^{2}\right) .
$$

Using this, the first inequality in Lemma 6.3 and (6.4) we see that

$$
\|\eta\|_{U_{1} \cap U_{2}}^{2} \leq c_{9}\left(\left\|\overline{\overline{\psi_{1}}}\right\|_{U_{1}}^{2}+\left\|\overline{\overline{\psi_{2}}}\right\|_{U_{2}}^{2}\right)
$$

Recall that we have fixed $r_{0}$ so that $\nu\left(R-r_{0}+1\right) \geq y^{2}$. We combine the second inequality in Lemma 6.3 and (6.2) to get

$$
c_{9} y^{2}\|\eta\|_{U_{1} \cap U_{2}}^{2} \leq\|\phi\|_{U_{1}}^{2}+\|\overline{\bar{\phi}}\|_{U_{2}}^{2} \text {. }
$$

This, together with (6.5), implies that

$$
c_{10} y^{2}\|\eta\|_{U_{2}}^{2} \leq\|\phi\|_{U_{1}}^{2}+\|\overline{\bar{\phi}}\|_{U_{2}}^{2} \leq 2\|\phi\|_{U_{1}}^{2}+\|\overline{\bar{\phi}}\|_{U_{2} \backslash U_{1}}^{2}
$$

To complete the proof of the estimate above we need to prove Lemma 6.3.

Proof of Lemma 6.3. Since $\overline{\overline{\psi_{1}}}-\overline{\overline{\psi_{2}}}=d g$ and $g$ is perpendicular to constants on every torus $F_{r}$, the first inequality follows as in [2, (3.8)]. The point here is that the torus $F_{r}$ shrinks at a definite rate as it recedes from the boundary of the tube, i.e. as $r$ decreases.

To prove the second inequality, recall that $\overline{\overline{\psi_{2}}}$ is coexact and $\overline{\overline{\phi_{2}}}=d \overline{\overline{\psi_{2}}}$. In particular, the normal component of $\overline{\overline{\psi_{2}}}$ on the boundary of $U_{2}$ vanishes. Therefore, if we write $\overline{\overline{\psi_{2}}}$ as $\overline{\overline{\psi_{2}}}=f d r+\alpha, f=0$ on $\partial U_{2}$. We now use (2.3) to estimate $\left(d \overline{\overline{\psi_{2}}}, d \overline{\overline{\psi_{2}}}\right)_{U_{2}}$. The last two terms in (2.3) cancel, and all other terms are nonnegative. Integration by parts along $F_{r}$ shows that

$$
\begin{aligned}
(\overline{\bar{\phi}}, \overline{\bar{\phi}})_{U_{2}} & =\left(d \overline{\overline{\psi_{2}}}, d \overline{\overline{\psi_{2}}}\right)_{U_{2}} \\
& \geq\left(d^{r} f, d^{r} f\right)_{U_{2}}+\left(d^{r} \alpha, d^{r} \alpha\right)_{U_{2}}+\left(\vartheta^{r} \alpha, \vartheta^{r} \alpha\right)_{U_{2}} \\
& =\left(\Delta^{r} \overline{\overline{\psi_{2}}}, \overline{\overline{\psi_{2}}}\right)_{U_{2}} .
\end{aligned}
$$

Since $F_{r}$ is a two-dimensional surface, the nonzero eigenvalues for forms and functions on it are equal, i.e. the first positive eigenvalue of the Laplacian $\Delta^{r}$ on forms of degree one on $F_{r}$ is equal to $\nu(r)$. Since $\nu(r)$ is increasing (cf. [2, Lemma 3.9] $),\left(\Delta^{r} \overline{\overline{\psi_{2}}}, \overline{\overline{\psi_{2}}}\right)_{U_{2}} \geq \nu\left(R-r_{0}+1\right)\left(\overline{\overline{\psi_{2}}}, \overline{\overline{\psi_{2}}}\right)_{U_{2}}$ which proves the lemma.

Finally, we construct a form $\kappa$ on $U_{2}$ with coefficients depending only on $r$ which is an extension of $\overline{\psi_{1}}$ to $U_{2}$ and satisfies $d \kappa=\bar{\phi}$. The forms $\overline{\psi_{1}}, \bar{\phi}$ can be written on $U_{1} \cap T$ as follows.

$$
\begin{aligned}
\overline{\psi_{1}} & =f d r+b_{1} d t+b_{2} d \theta, \\
\bar{\phi} & =a_{1} d r \wedge d t+a_{2} d r \wedge d \theta .
\end{aligned}
$$

Therefore

$$
b_{i}^{\prime}=a_{i}
$$

on $U_{1} \cap T$, i.e. for $r \in\left[R-r_{0}, r\right]$.

We extend $b_{i}$ to the interval $\left[0, R-r_{0}\right]$ so that these equations continue to hold. The extension is given for $r>0$ by

$$
b_{i}(r)=b_{i}\left(R-r_{0}\right)-\int_{r}^{R-r_{0}} a_{i}(\rho) d \rho .
$$


We then set

$$
\kappa=h(r) f(r) d r+b_{1}(r) d t+b_{2}(r) d \theta
$$

with a cut-off function $h(r)$ equal to one for $r \leq R-r_{0}$, equal to zero for $r \geq R-r_{0}+1$ and linear in $\left[R-r_{0}+1, R-r_{0}\right]$. Obviously $d \kappa=\phi$ on $U_{2} \backslash \gamma$. We have to check that $\kappa$ belongs to the domain of $d$, i.e. that it is in $L^{2}$ and its distributional derivative is equal to $\phi$. There is no difficulty with $b_{1} d t$ since it is smooth on $T \backslash \gamma$ and continuous on $T$. However $\|d \theta\|_{\infty}=O(1 / r)$ for small $r$. If we require that

$$
\int_{0}^{R-r_{0}} a_{2}(\rho) d \rho=b_{2}\left(R-r_{0}\right),
$$

then $b_{2}(r)=O(r)$ for small $r$ so that $b_{2}(r) d \theta$ is bounded and has bounded exterior derivative $a_{2} d r \wedge d \theta$ on $T \backslash \gamma$. This is sufficient regularity for our purposes. We add (6.10) to our list of conditions on $\phi$.

We still need to estimate the $L^{2}$ norm of $\kappa$, i.e.

$$
2 \pi l \int_{0}^{R-r_{0}} b_{1}^{2} \tanh \rho d \rho+2 \pi l \int_{0}^{R-r_{0}} b_{2}^{2} \operatorname{coth} \rho d \rho
$$

in terms of the $L^{2}$ norm of $d \kappa=\phi$, i.e.

$$
2 \pi l \int_{0}^{R-r_{0}}\left(b_{1}^{\prime}\right)^{2} \tanh \rho d \rho+2 \pi l \int_{0}^{R-r_{0}}\left(b_{2}^{\prime}\right)^{2} \operatorname{coth} \rho d \rho .
$$

What we need are the following inequalities.

$$
\begin{aligned}
& \frac{\int_{0}^{R-r_{0}}\left(b_{1}^{\prime}\right)^{2} \tanh \rho d \rho}{\int_{0}^{R-r_{0}} b_{1}^{2} \tanh \rho d \rho} \geq x^{2}, \\
& \frac{\int_{0}^{R-r_{0}}\left(b_{2}^{\prime}\right)^{2} \operatorname{coth} \rho d \rho}{\int_{0}^{R-r_{0}} b_{2}^{2} \operatorname{coth} \rho d \rho} \geq x^{2},
\end{aligned}
$$

where $x$ is the parameter in (1.1). Note that the expressions on the left-hand sides are the Rayleigh-Ritz quotients (3.1) and (3.2).

This of course can be true only after imposing additional conditions. To see what kind and how many conditions are needed we investigate the quadratic forms defined by the numerators of the Rayleigh-Ritz quotients above. Formal integration by parts yields two ordinary differential operators

$$
L_{1} u=-\frac{1}{\tanh r}\left(u^{\prime} \tanh r\right)^{\prime}, \quad L_{2} v=-\frac{1}{\operatorname{coth} r}\left(v^{\prime} \operatorname{coth} r\right)^{\prime}
$$

already considered in Section 3. We will investigate the spectra of these operators for appropriate boundary conditions. To simplify matters we impose two additional conditions on $\phi$. Namely we require that $b_{i}\left(R-r_{0}\right)=0$ for $i=1,2, r=0$ is a regular singular point for both $L_{1}$ and $L_{2}$. We consider the two operators separately. Classical analysis (Frobenius series) shows that $L_{1}$ is in the limit circle case, i.e. the equation $L_{1} u=0$ has two linearly independent solutions which are in $L^{2}(\tanh r d r)$. One of these is bounded near $r=0$ while the other has a logarithmic singularity. Since in our case $b_{1}$ is bounded, we study the eigenvalue problem

$$
L_{1} u=\nu u, \quad u\left(R-r_{0}\right)=0, \quad u(r)=O(1) \text { near } 0 .
$$


The second operator turns out to be in the limit point case, i.e. up to a multiplicative factor there is only one $L^{2}(\operatorname{coth} r d r)$ solution of the equation $L_{2} v=0$. All $L^{2}$ solutions are $O\left(r^{2}\right)$ at zero. Thus, the appropriate eigenvalue problem for $L_{2}$ is

$$
L_{2} v=\mu v, \quad v\left(R-r_{0}\right)=0, \quad v=O\left(r^{2}\right) \text { near } 0 .
$$

We use the substitutions $u=\tanh ^{-1 / 2} r f$ for $L_{1}$ and $v=\operatorname{coth}^{-1 / 2} r g$ for $L_{2}$ to transform the two Sturm-Liouville problems to a standard form. The two problems become

$$
\begin{gathered}
-f^{\prime \prime}-\frac{1+4 \sinh ^{2} r}{\sinh ^{2}(2 r)} f=\nu f, \quad f\left(R-r_{0}\right)=0, \\
f=O\left(r^{1 / 2}\right) \text { near } 0,
\end{gathered}
$$

and

$$
\begin{gathered}
-g^{\prime \prime}+\frac{4 \cosh ^{2} r-1}{\sinh ^{2}(2 r)} g=\mu g, \quad g\left(R-r_{0}\right)=0, \\
g=O\left(r^{3 / 2}\right) \text { near } 0,
\end{gathered}
$$

respectively.

The first of these problems has discrete spectrum since according to [11, 5.12] this is true for any choice of selfadjoint boundary conditions in the limit circle case. The second problem has discrete spectrum as well since the potential $\left(4 \cosh ^{2} r-1\right) / \sinh ^{2}(2 r)$ tends to positive infinity at 0 (cf. $\left.[11,5.15]\right)$. This shows that the singular point at $r=0$ causes no difficulties. In particular, each. of the two problems admits a complete orthonormal system of eigenfunctions and all consequences of the min-max characterization of eigenvalues hold. For example, it is easy to show using Dirichlet-Neumann bracketing that for large $R$ the number of eigenvalues of either problem in the interval $\left[0, x^{2}\right]$ differs by a finite number bounded independently of $R$ from the number of eigenvalues in $\left[0, x^{2}\right]$ of the Dirichlet problem for the same operator on $\left[1, R-r_{0}\right]$. Theorem 4.1 of [2] applies therefore to (6.15) and (6.16) so that the number of eigenvalues below $x^{2}$ for either problem is equal to $x R / \pi+O_{x}(1)$.

Now, if the form $\phi$ considered at the outset is such that the coefficients $b_{1}$ and $b_{2}$ determined by it are perpendicular in appropriate weighted $L^{2}$ spaces to all eigenfunctions with eigenvalues in $\left[0, x^{2}\right)$ of $(6.13)$ and (6.14) respectively, then both (6.11) and (6.12) hold. This follows from variational characterization of the eigenvalues of the two Sturm-Liouville problems and is equivalent to

$$
x^{2}(\kappa, \kappa)_{U_{2} \backslash U_{1}} \leq(\bar{\phi}, \bar{\phi})_{U_{2} \backslash U_{1}} .
$$

We have thus constructed a form $\psi$ of degree one on $M$ which satisfies $d \psi=\phi$. To recapitulate

$$
\psi\left|U_{1} \backslash U_{2}=\psi_{1}, \quad \psi\right| U_{2}=\eta+\kappa .
$$

By construction, $\eta=\overline{\bar{\eta}}$ and $\kappa=\bar{\kappa}$. Since the summands in (6.1) are orthogonal on every torus $F_{r}, \eta$ and $\kappa$ are orthogonal to each other in $L^{2} A^{1}\left(U_{2}\right)$. Set $y=2 x \max \left\{1, c_{10}^{-1 / 2}\right\}$, where $c_{10}$ is the constant appearing in (6.6). It follows from (6.2) that

$$
x^{2}\left(\psi_{1}, \psi_{1}\right)_{U_{1} \backslash U_{2}} \leq \frac{1}{4}\left(\phi_{1}, \phi_{1}\right)_{U_{1}} .
$$


Similarly, (6.6) implies that

$$
x^{2}(\eta, \eta)_{U_{2}} \leq \frac{1}{2}(\phi \phi)_{U_{1}}+(\overline{\bar{\phi}}, \overline{\bar{\phi}})_{U_{2} \backslash U_{1}} .
$$

From the definition of $\kappa$ and (6.2) we obtain

$$
x^{2}(\kappa, \kappa)_{U_{1} \cap U_{2}} \leq \frac{1}{4}(\phi, \phi)_{U_{1}} .
$$

Adding the three inequalities above to $(6.17)$ we get

$$
x^{2}(\psi, \psi)_{M} \leq(\phi, \phi)_{M}
$$

The number of linear conditions $C$ which we had to impose during the construction was $2 x R / \pi$ up to a bounded error which depends on $x$ but not on $R$. We apply Lemma 4.2 with $m=C+1$ to conclude that $\lambda_{C+1} \geq x^{2}$. This proves that

$$
N(x) \leq 2 \frac{x R}{\pi}+O_{x}(1)
$$

which together with (3.3) yields (1.1).

\section{REFERENCES}

1. R. Bott and L. W. Tu, Differential forms in algebraic topology, Graduate Texts in Math., no. 82, Springer-Verlag, New York, 1982.

2. I. Chavel and J. Dodziuk, The spectrum of degenerating hyperbolic manifolds of three dimensions, J. Differential Geom. 39 (1994), 123-171.

3. J. Cheeger, On the Hodge theory of Riemannian pseudomanifolds, Geometry of the Laplace Operator (R. Osserman and A. Weinstein, eds.), Proc. Sympos. Pure Math., vol. 36, Amer. Math. Soc., Providence, RI, 1980, pp. 91-146.

4. _ـ Spectral geometry of singular Riemannian spaces, J. Differential Geom. 18 (1983), 575-657.

5. M. Gromov, Hyperbolic manifolds (according to Thurston and Jørgensen), Seminaire Bourbaki \#546, 1979, Lecture Notes Notes in Math., vol. 842, Springer, 1981, pp. 40-53.

6. D. Kazhdan and G. Margulis, A proof of Selberg's hypothesis, Mat. Sb. 75 (117) (1968), 163-168.

7. R. Mazzeo and R. Phillips, Hodge theory on hyperbolic manifolds, Duke Math. J. 60 (1990), 509-559.

8. J. McGowan, The p-spectrum of compact hyperbolic three manifolds, Math. Ann. 279 (1993), 725-745.

9. L. E. Payne and H. F. Weinberger, An optimal Poincaré inequality for convex domains, Arch. Rational Mech. Anal. 5 (1960), 286-292.

10. W. Thurston, The geometry and topology of 3-manifolds, Department of Mathematics, Princeton Univ., Princeton, NJ, 1980.

11. E. C. Titchmarch, Eigenfunction expansions associated with second order differential equations, Vol. 1, Cambridge Univ. Press, London, 1946.

Graduate School and University Center (CUNY), 33 West 42Nd Street, New York, NEW YORK 10036-8099

E-mail address: jzdqc@cunyvm. cuny.edu

Department of Mathematics, Central Connecticut State University, New Britain, CONNECTICUT 06050

E-mail address: mcgowan@ctstateu.edu 